\title{
A Comparison Study of the Shannon Channel Capacity of Various Nonlinear Optical Fibers
}

\author{
Jau Tang
}

\begin{abstract}
A comparative study of the Shannon channel capacity is presented for a dispersion-free fiber, a fiber with constant dispersion, and a fiber with variable dispersion. Improvement of the capacity by optical phase conjugation (OPC) is also investigated. Simple scaling laws are prescribed for the dependence of the optimal capacity on various system settings such as number of spans, number of channels, noise power, channel width, strength of chromatic dispersion, bandwidth of an OPC device, etc.
\end{abstract}

Index Terms-Fiber nonlinearity, information theory, optical fiber communication systems, optical phase conjugation, Volterra series, wavelength division multiplexing systems.

\section{INTRODUCTION}

$\mathbf{T}$ HE ULTIMATE channel capacity of a nonlinear optical fiber is a fundamental question. It is important to know its theoretical limit and the controlling factors for capacity degradation for better design of next-generation transmission systems. According to the well-known Shannon's theory [1], for a linear transmission system with a signal-to-noise ratio $S / N$, the optimal channel capacity is given by $\log _{2}(1+S / N)$. For a typical optical amplifier with an $S / N$ ratio of $40 \mathrm{~dB}$, one would expect a theoretical limit for the channel capacity of about $13 \mathrm{bits} / \mathrm{s} / \mathrm{Hz}$ if Kerr nonlinear effects such as selfphase modulation, cross-phase modulation, and four-wave mixing are absent. To investigate the capacity degradation due to these nonlinear effects, in a series of reports we presented the Shannon channel capacity calculation for a dispersion-free fiber (single span [2] and multispan cases [3]) and a fiber with constant dispersion [4]. In these studies, we consider a transmission system with ideal coherent detection. At the end of each span, the optical signal is amplified to compensate for the fiber attenuation and white Gaussian amplifier noise is added. These studies indicate a theoretical capacity of about one order of magnitude higher than that has been achieved by current technologies in dense wavelength division multiplexed (DWDM) systems.

The previous capacity calculation, particularly for dispersive fibers, relies on numerical computation involving multidimensional integrals [4]. In this work, it is shown that these integrals can be expressed in an approximate closed form. Analytical formulae are given for the ultimate channel capacity and the optimal input power for three categories of fibers. Simple scaling laws are prescribed for the dependence of the ulti-

Manuscript received October 26, 2005; revised January 12, 2006.

The author was with Lucent Technologies, Holmdel, NJ 07733 USA. He is now with Noyes Laboratories, California Institute of Technology, Pasadena, CA 91125 USA (e-mail: jautang@ caltech.edu).

Digital Object Identifier 10.1109/JLT.2006.872293 mate capacity and the corresponding optimal operating input power on various system settings. These settings include the number of channels, number of spans, noise power, channel width, strength of chromatic dispersion, bandwidth of an optical phase conjugation (OPC) device, etc. This study also includes consideration of nonlinearity precompensation using interchannel or intrachannel OPC [5], [6] to eliminate some nonlinear noise. The extent of capacity improvement by OPC and the dependence of capacity improvement on the bandwidth of an OPC device will be analyzed. This work supplements channel capacity studies by others [7]-[9] using a different approach.

\section{Volterra Series SOlUtion fOR THE NONLINEAR SCHROEDINGER EQUATION (NSE)}

The NSE for waveform propagation in a nonlinear fiber is given by [10]

$$
\frac{\partial A(z, t)}{\partial z}=\hat{D}(z) A(z, t)+i \gamma|A(z, t)|^{2} A(z, t)
$$

where $2 \hat{D}(z)=-\alpha+i \beta(z) \partial^{2} / \partial t^{2}$. The dispersion $\beta(z)$ can be a constant or length dependent. In a variable dispersion fiber, the dispersion is designed to match the attenuation in power, i.e., $\beta(z)=\beta e^{-\alpha z}$. For a dispersion-free case with $\beta=0$, the exact solution of NSE is given by $A(L, t)=$ $\exp (-\alpha L / 2) \exp \left(i \gamma_{L}|A(t)|^{2}\right) A(t)$, and $\gamma_{L}$ is defined as $\gamma[1-$ $\exp (\alpha L)] / \alpha$, where $A(t)$ is the input signal, $\alpha$ the absorption coefficient, and $\gamma$ the Kerr nonlinearity coefficient. For a dispersive fiber, one can solve NSE by Volterra series expansion [11] in terms of the Kerr nonlinearity, i.e., $A(z, t)=$ $\sum_{k=0}^{\infty} \gamma^{k} A_{k}(z, t)$. The first two terms are

$$
\begin{aligned}
& \frac{\partial A_{0}(z, t)}{\partial z}=\hat{D}(z) A_{0}(z, t) \\
& \frac{\partial A_{1}(z, t)}{\partial z}=\hat{D}(z) A_{1}(z, t)+i A_{0}(z, t) A_{0}^{*}(z, t) A_{0}(z, t) .
\end{aligned}
$$

For convenience, we use the following short-hand expression as the formal solution, i.e., $A(L, t)=\exp (-\alpha L / 2) \boldsymbol{U} A(t)$, where $\boldsymbol{U}$ is a nonlinear operator that is a function of $A(t)$. For a multispan system, at the end of each span, the signal is amplified by a factor of $\exp (\alpha L / 2)$ to compensate the power attenuation. The amplified output signal after $N$ spans and $N$ amplifiers, which is defined as $A(N L, t)$, can be given by a recursive formula as $\exp \left(i \gamma_{L}|A((N-1) L, t)|^{2}\right) A((N-$ 1) $L, t)+\sigma_{N}(t)$, where $\sigma_{N}(t)$ is the noise from the $N$ th amplifier. By iteration, one can express $A(N, t)$ in terms of the input signal $A(t)$ and noise signals $\sigma_{1}(t), \sigma_{2}(t), \ldots, \sigma_{N}(t)$, 
from all preceding amplifiers. As an example, for a two-span system, one has $A(2 L, t)=\boldsymbol{U} A(L, t)+\sigma_{2}(t)=\boldsymbol{U}[\boldsymbol{U} A(t)+$ $\left.\sigma_{1}(t)\right]+\sigma_{2}(t)$.

Using the nonlinearity precompensation procedure with OPC, one essentially reverses the nonlinear phase for one or a few channels, depending on the bandwidth of such a device. If each channel is precompensated, one prepares $\boldsymbol{U}_{\boldsymbol{n}}^{-1} A_{n}(t)$ for the $n$th channel before one combines all channels and feeds it into the transmission fiber. The output signal after one span is given by $A(L, t)=\exp (-\alpha L / 2) \boldsymbol{U}\left(\sum_{n} \boldsymbol{U}_{n}^{-1} A_{n}(t)\right)$, where $\sum_{n}$ denotes the summation over $n$ channels. In this work, we will consider both schemes for various transmission fibers with/without precompensation.

\section{SHANNON CAPACITY (SC) FOR A NONLINEAR TRANSMISSION SYSTEM}

As discussed in an earlier work [2]-[4], one can use the conventional Shannon's information theory to a nonlinear system by applying Pinsker's generalized capacity formula [12]. For an $N$-span system, we have

$$
\mathrm{SC}=\frac{1}{\omega_{c}} \int_{-\frac{\omega_{c}}{2}}^{\frac{\omega_{c}}{2}} d \omega \log _{2}\left(\frac{f(\omega)}{g(\omega)}\right)
$$

where $g(\omega)$ and $f(\omega)$ are given by a determinant

$$
\begin{aligned}
& g(\omega)=\left|\begin{array}{cc}
F\left(\left\langle A(t+\tau) A^{*}(t)\right\rangle\right) & F\left(\left\langle A(N, t+\tau) A^{*}(t)\right\rangle\right) \\
F\left(\left\langle A(t+\tau) A^{*}(N, t)\right\rangle\right) & F\left(\left\langle A(N, t+\tau) A^{*}(N, t)\right\rangle\right)
\end{array}\right| \\
& f(\omega)=\left|\begin{array}{cc}
F\left(\left\langle A(t+\tau) A^{*}(t)\right\rangle\right) & 0 \\
0 & F\left(\left\langle A(N, t+\tau) A^{*}(N, t)\right\rangle\right)
\end{array}\right|
\end{aligned}
$$

where $F$ stands for Fourier transform. The covariant determinant is needed [12] because the output signal $A(N L, t)$ is highly correlated with the input $A(t)$ in a nonlinear transmission system. For the dispersion-free case, one can obtain a closed-form expression for the above correlation function in terms of input function [2], [3]. However, for a dispersive fiber, solving the NSE analytically and evaluating the above correlation function are almost impossible. In the limit of low input power, we considered only two lowest perturbation terms. For a single-span system, (1) can be approximated by

$$
\begin{aligned}
g(\omega) \approx & F\left[\left\langle e^{\alpha L} A(t+\tau) A^{*}(t)\right\rangle\right] \\
& \times F\left[\left\langle e^{\alpha L} A_{1}(N L, t+\tau) A_{1}^{*}(N L, t)\right\rangle\right] \\
& -F\left[\left\langle e^{\alpha L} A(t+\tau) A_{1}^{*}(N L, t)\right\rangle\right] \\
& \times F\left[\left\langle e^{\alpha L} A_{1}(N L, t+\tau) A^{*}(t)\right\rangle\right] \\
& +P_{W}(\omega) F\left[\left\langle e^{\alpha L} A(t+\tau) A^{*}(t)\right\rangle\right] \\
f(\omega) \approx & F\left[\left\langle e^{\alpha L} A(t+\tau) A^{*}(t)\right\rangle\right] \\
& \times F\left[\left\langle A(t+\tau) A^{*}(t)\right\rangle\right]+g(\omega)
\end{aligned}
$$

where $P_{W}(\omega)$ is the white noise power density. For an $N$ span system, given the fact of very low noises in the optical amplifier, one can exclude the nonlinear effects from the noises themselves. As long as the nonlinear effects are dominated by the input signal, one can treat the multispan system as an effective single-span system but with noise power and nonlinear coupling correctly scaled up. For dispersion-free fibers, one only needs to scale the effective Kerr nonlinear coupling and the noise power density by $N$ times. However, for a dispersive fiber (with $\beta$ greater than $0.5 \mathrm{ps}^{2} / \mathrm{km}$ and a total bandwidth of more than $1 \mathrm{THz}$ ), one needs to scale the effective Kerr nonlinear coupling by $N^{1 / 2}$ and the noise power density by $N$ times. A large dispersion reduces the correlation length and makes the nonlinear noises from each span quasi-independent. In the following three sections, the analysis of SC for each category of fibers will be presented in an individual section.

\section{SC FOR A DISPERSION-FREE FIBER}

In our model calculation, we assume a flat spectral distribution for $N_{c}$ equally wide channels, each with a width of $\omega_{c}$. The total bandwidth is $\Omega_{T}=N_{c} \omega_{c}$. For a multispan ( $N_{S}$ spans) system, we have shown [2] that both the noise power density and the Kerr nonlinear coupling $\gamma$ have to be scaled by $N_{S}$. For the dispersion-free case, $g(\omega)$ and $f(\omega)$ in (4) can be calculated to any power expansion of $\gamma$ as

$$
\begin{aligned}
g(\omega) \approx & N_{S} P_{W}(\omega) \\
& +\frac{2 N_{S}^{2} \gamma_{L}^{2}}{\left[1+\gamma_{L}^{2} N_{S}^{2} \Omega_{T}^{2}\left(\frac{P}{2 \pi \omega_{c}}\right)^{2}\right]^{3}} F\left(R(\tau)|R(\tau)|^{2}\right) \\
& +\frac{3 N_{S}^{4} \gamma_{L}^{4}}{\left[1+\gamma_{L}^{2} N_{S}^{2} \Omega_{T}^{2}\left(\frac{P}{2 \pi \omega_{c}}\right)^{2}\right]^{4}} F\left(R(\tau)|R(\tau)|^{4}\right)+\ldots \\
f(\omega) \approx & \frac{\frac{P}{\omega_{c}}}{1+\gamma_{L}^{2} N_{S}^{2} \Omega_{T}^{2}\left(\frac{P}{2 \pi \omega_{c}}\right)^{2}}+g(\omega)
\end{aligned}
$$

where $R(\tau)$ is the autocorrelation of the input signal, and $P$ is the input power per channel with channel width $\omega_{c}$. Assuming a flat spectral distribution with total bandwidth $\Omega_{T}$ for the input signal, $R(\tau)=\left(P / \pi \omega_{c} \tau\right) \sin \left(\Omega_{T} \tau / 2\right)$. Here, $\gamma_{L}$ is defined as $\gamma\left[1-\exp \left(-\alpha_{L}\right)\right] / \alpha$. For two other kinds of fibers, $f(\omega)$ is given by the same expression as above.

Although $g(\omega)$ in (6) can be evaluated for any power of $\gamma$, in practical applications with weak power, one only needs to keep the lowest order term and

$$
\begin{aligned}
& g(\omega) \approx N_{S} P_{W}(\omega)+\frac{3}{2} \\
& \times \frac{N_{S}^{2} \alpha^{2} \gamma_{L}^{2}}{\left[1+\gamma_{L}^{2} N_{S}^{2} \Omega_{T}^{2}\left(\frac{P}{2 \pi \omega_{c}}\right)^{2}\right]^{3}}\left(\frac{\Omega_{T}}{2 \pi}\right)^{2}\left(\frac{P}{\omega_{c}}\right)^{3}\left(1-\frac{\omega^{2}}{3 \Omega_{T}^{2}}\right) .
\end{aligned}
$$

The Kerr nonlinear noises and the white noises from the amplifier contribute to $g(\omega)$. From (1), the SC for the central channel 
can be approximated by

$$
\left.\mathrm{SC} \approx \log _{2}\left[1+\frac{\frac{P}{\omega_{c}}}{\frac{3}{8 \pi^{2}} \frac{N_{S}^{2} \gamma_{L}^{2} \Omega_{T}^{2}}{\left[1+\gamma_{L}^{2} N_{S}^{2} \Omega_{T}^{2}\left(\frac{P}{2 \pi \omega_{c}}\right)^{2}\right]^{3}}\left(\frac{P}{2 \pi \omega_{c}}\right)^{2}}\right)^{3}+N_{S} P_{W}\right]
$$

where $P$ is the input power density. From (8), one can determine the maximum capacity $\mathrm{SC}_{\mathrm{MAX}}$ and the optimal operating input power per channel $P_{\text {MAX }}$ for a dispersion-free fiber as

$$
\begin{aligned}
\mathrm{SC}_{\mathrm{MAX}} & \approx \log _{2}\left[1+\left(\frac{32 \pi^{2}}{81}\right)^{\frac{1}{3}}\left(N_{S}^{2} N_{c} \gamma_{L} P_{W} \omega_{c}\right)^{-\frac{2}{3}}\right] \\
P_{\mathrm{MAX}} & \approx\left(\frac{4 \pi^{2}}{3}\right)^{\frac{1}{3}}\left(N_{S} N_{c}^{2} \gamma_{L}^{2}\right)^{-\frac{1}{3}}\left(P_{W} \omega_{c}\right)^{\frac{1}{3}} .
\end{aligned}
$$

The second term in $P_{\mathrm{MAx}}$ is a small correction and may be neglected for weak amplifier noise power. The above scaling law of (9) was reported in our earlier work [4]. The new result presented here is that it precisely prescribes the proportional constant that was unspecified previously.

Now, one can examine the nonlinearity precompensation case. Kerr nonlinear noises can be reduced by a nonlinearity precompensation procedure with an OPC device. Assuming such a device has a bandwidth of $\Omega_{\mathrm{OPC}}$ so that several channels can be compensated all together, a revised $g(\omega)$ is given by

$$
\begin{aligned}
g(\omega) \approx & N_{S} P_{W}(\omega)+\frac{3}{8 \pi^{2}} \frac{N_{S}^{2} \gamma_{L}^{2}}{\left[1+\gamma_{L}^{2} N_{S}^{2} \Omega_{T}^{2}\left(\frac{P}{2 \pi \omega_{c}}\right)^{2}\right]^{3}}\left(\frac{P}{\omega_{c}}\right)^{3} \\
\times & \left\{\Omega_{T}^{2}\left(1-\frac{\omega^{2}}{3 \Omega_{T}^{2}}\right)-\sum_{n} \Omega_{\mathrm{OPC}}^{2}\left[1-\frac{\left(\omega-n \omega_{c}\right)^{2}}{3 \Omega_{\mathrm{OPC}}^{2}}\right]\right. \\
& \left.\times\left[\eta\left(\omega-n \omega_{c}+\Omega_{\mathrm{OPC}}\right)-\eta\left(\omega-n \omega_{c}-\Omega_{\mathrm{OPC}}\right)\right]\right\}
\end{aligned}
$$

where $\eta(x)$ is a step function such that $\eta(x)=0$ if $x$ is negative and 1 otherwise. If OPCs with a bandwidth of $\Omega_{\mathrm{OPC}}$ were applied, one has the capacity for the central channel $(n=0)$ as that shown in (10b) at the bottom of the page. In this work, $\Omega_{\mathrm{OPC}}$ is assumed to be smaller than $\Omega_{T}$. If $\Omega_{\mathrm{OPC}}$ is larger than
$\Omega_{T}$, the nonlinear noises are eliminated, and one should use the classical Shannon formula. One has

$$
\begin{aligned}
& \mathrm{SC}_{\mathrm{MAX}} \approx \log _{2} {\left[1+\left(\frac{32 \pi^{2}}{81}\right)^{\frac{1}{3}}\right.} \\
&\left.\times\left(N_{S}^{2} \gamma_{L} P_{W} \omega_{c}\left(N_{c}^{2}-\frac{\Omega_{\mathrm{OPC}}^{2}}{\omega_{c}^{2}}\right)^{\frac{1}{2}}\right)^{-\frac{2}{3}}\right] \\
& P_{\mathrm{MAX}} \approx\left(\frac{4 \pi^{2}}{3}\right)^{\frac{1}{3}}\left[N_{S} \gamma_{L}^{2}\left(N_{c}^{2}-\frac{\Omega_{\mathrm{OPC}}^{2}}{\omega_{c}^{2}}\right)\right]^{-\frac{1}{3}}\left(P_{W} \omega_{c}\right)^{\frac{1}{3}}
\end{aligned}
$$

\section{SC FOR FibERS With A CONSTANT Dispersion}

For a constant dispersion, one can determine the solution for the first two terms of the Volterra series in (2). The scaling factor for the effective $\gamma$ in a multispan system here is the square root of the total number of span [4] and is different from the dispersion-free case [3]. We have obtained an explicit expression for the corresponding $g(\omega)$ and $f(\omega)$ in (2) as

$$
\begin{aligned}
g(\omega) \approx N_{S} P_{W}(\omega) & +\frac{2 N_{S}}{\left[1+N_{s}^{2} \gamma_{L}^{2} \Omega_{T}^{2}\left(\frac{P}{2 \pi \omega_{c}}\right)^{2}\right]^{3}}\left(\frac{\gamma}{2 \pi}\right)^{2} \\
& \times \int_{-\infty}^{\infty} d \omega_{1} \int_{\infty}^{\infty} d \omega_{2} \frac{1+e^{-2 \alpha L}-2 e^{-\alpha L} \cos \left[L \beta\left(\omega-\omega_{1}\right)\left(\omega_{1}-\omega_{2}\right)\right]}{\alpha^{2}+\beta^{2}\left(\omega-\omega_{1}\right)^{2}\left(\omega_{1}-\omega_{2}\right)^{2}} \\
& \times R\left(\omega_{1}\right) R\left(\omega_{2}\right) R\left(\omega-\omega_{1}+\omega_{2}\right) .
\end{aligned}
$$

In our previous work [4], the numerical approach was used to carry out the two-dimensional (2-D) integral. For most dispersive fibers, we have derived a very good approximate analytical expression for the 2-D integral. Our analytical approximated expression for $g(\omega)$ and $f(\omega)$ is given by

$$
\begin{aligned}
& g(\omega) \approx N_{S} P_{W}(\omega) \\
& +\frac{N_{S} \gamma_{L}^{2}}{\left[1+\gamma_{L}^{2} N_{s}^{2} \Omega_{T}^{2}\left(\frac{P}{2 \pi \omega_{c}}\right)^{2}\right]^{3}}\left(\frac{P}{\omega_{c}}\right)^{3} \frac{\alpha}{\pi \beta} \ln \left[\frac{\beta N_{c}^{2} \omega_{c}^{2}}{4 \alpha}\right] .
\end{aligned}
$$

The above result is valid for $\beta \Omega_{T}^{2} / \alpha \gg 10$ and $\exp (-\alpha L) \ll$ 1. For $\alpha=0.048 \mathrm{~km}^{-1}, \beta=1 \mathrm{ps}^{2} / \mathrm{km}$, and $\Omega_{T} / 2 \pi=1 \mathrm{THz}$, these conditions are well satisfied. The SC for the central

$$
\mathrm{SC}_{\mathrm{OPC}} \approx \log _{2}\left[1+\frac{\frac{\frac{P}{\omega_{c}}}{1+\gamma_{L}^{2} N_{S}^{2} \Omega_{T}^{2}\left(\frac{P}{2 \omega_{c}}\right)^{2}}}{\frac{3}{8 \pi^{2}} \frac{N_{S}^{2} \alpha^{2} \gamma_{L}^{2}}{\left[1+\gamma_{L}^{2} N_{S}^{2} \Omega_{T}^{2}\left(\frac{P}{2 \pi \omega_{c}}\right)^{2}\right]^{3}}\left(\Omega_{T}^{2}-\Omega_{\mathrm{OPC}}^{2}\right)\left(\frac{P}{\omega_{c}}\right)^{3}+N_{S} P_{W}}\right]
$$


channel is given as that shown in (14) at the bottom of the page. The maximum capacity and the optimal operating input power per channel are

$$
\begin{aligned}
\mathrm{SC}_{\mathrm{MAX}} \approx \log _{2}[1 & +\frac{2}{3} N_{s}^{-1}\left(\gamma_{L} P_{W} \omega_{c}\right)^{-\frac{2}{3}} \\
& \left.\times\left(\frac{2 \alpha}{\pi \beta \omega_{c}^{2}} \ln \left[\frac{\beta N_{c}^{2} \omega_{c}^{2}}{4 \alpha}\right]\right)^{-\frac{1}{3}}\right] \\
P_{\mathrm{MAX}} \approx & {\left[\frac{2 \alpha \gamma_{L}^{2}}{\pi \beta \omega_{c}^{2}} \ln \left(\frac{\beta N_{c}^{2} \omega_{c}^{2}}{4 \alpha}\right)\right]^{-\frac{1}{3}}\left(P_{W} \omega_{c}\right)^{\frac{1}{3}} . }
\end{aligned}
$$

We can now consider the case with nonlinearity precompensation. If OPCs with a bandwidth of $\Omega_{\mathrm{OPC}}$ were applied, one has the capacity for the central channel $(n=0)$ as that shown in (16) at the bottom of the page. The maximum capacity and the optimal operating input power per channel are

$$
\begin{aligned}
& \mathrm{SC}_{\mathrm{MAX}} \approx \log _{2} {\left[1+\frac{2}{3}\left(\sqrt{N_{S}} \gamma_{L} P_{W} \omega_{c}\right)^{-\frac{2}{3}}\right.} \\
&\left.\times\left(\frac{2 \alpha}{\pi \beta \omega_{c}^{2}} \ln \left[\frac{N_{c}^{2} \omega_{c}^{2}}{\Omega_{\mathrm{OPC}}^{2}}\right]\right)^{-\frac{1}{3}}\right] \\
& P_{\mathrm{MAX}} \approx\left[\frac{2 \alpha \gamma_{L}^{2}}{\pi \beta \omega_{c}^{2}} \ln \left(\frac{N_{c}^{2} \omega_{c}^{2}}{\Omega_{\mathrm{OPC}}^{2}}\right)\right]^{-\frac{1}{3}}\left(P_{W} \omega_{c}\right)^{\frac{1}{3}}
\end{aligned}
$$

\section{SC FOR FIBERS WITH A LENGTH-DEPENDENT DISPERSION}

Now, we can consider the third category of fibers. In these fibers with a variable dispersion, the length-dependent dispersion is designed to match the power attenuation, i.e., $\beta(z)=$ $\beta \exp (-\alpha z)$. One can solve the NSE for the first two Volterra series in (2) by changing variable $y=\exp (-\alpha z)$. We have obtained the explicit expression for $g(\omega)$ and $f(\omega)$ as

$$
\begin{aligned}
& g(\omega) \approx N_{S} P_{W}(\omega)+\frac{1}{\pi^{2}} \frac{N_{S} \gamma^{2}}{\left[1+\gamma_{L}^{2} N_{s}^{2} \Omega_{T}^{2}\left(\frac{P}{2 \pi \omega_{c}}\right)^{2}\right]^{3}} \\
& \times \int_{-\infty}^{\infty} d \omega_{1} \int_{-\infty}^{\infty} d \omega_{2} \frac{1-\cos \left[\frac{\left(1-e^{-\alpha L}\right) \beta\left(\omega-\omega_{1}\right)\left(\omega_{1}-\omega_{2}\right)}{\alpha}\right]}{\beta^{2}\left(\omega-\omega_{1}\right)^{2}\left(\omega_{1}-\omega_{2}\right)^{2}} \\
& \times R\left(\omega_{1}\right) R\left(\omega_{2}\right) R\left(\omega-\omega_{1}+\omega_{2}\right) .
\end{aligned}
$$

For most practical fibers with nonzero dispersion, if $\beta \Omega_{T}^{2} / \alpha \gg$ 10 , the 2-D integral in $g(\omega)$ can be approximated by

$$
\begin{aligned}
& g(\omega) \approx N_{S} P_{W}(\omega)+\left(\frac{P}{\omega_{c}}\right)^{3} \\
& \times \frac{2 N_{S} \gamma_{L}^{2}}{\left[1+\gamma_{L}^{2} N_{s}^{2} \Omega_{T}^{2}\left(\frac{P}{2 \pi \omega_{c}}\right)^{2}\right]^{3}} \frac{\alpha}{\pi \zeta \beta}\left(\ln \left[\frac{\zeta \beta N_{c}^{2} \omega_{c}^{2}}{\alpha}\right]-1\right)
\end{aligned}
$$

where $\zeta=1-\exp (-\alpha L)$. The SC for the central channel is given as that shown in (20) at the bottom of the page. The

$$
\mathrm{SC} \approx \log _{2}\left(1+\frac{\frac{\frac{P}{\omega_{c}}}{1+\gamma_{L}^{2} N_{s}^{2} \Omega_{T}^{2}\left(\frac{P}{2 \pi \omega_{c}}\right)^{2}}}{\frac{N_{S} \gamma_{L}^{2}}{\left[1+\gamma_{L}^{2} N_{s}^{2} \Omega_{T}^{2}\left(\frac{P}{2 \pi \omega_{c}}\right)^{2}\right]^{3}}\left(\frac{P}{\omega_{c}}\right)^{3} \frac{\alpha}{\pi \beta} \ln \left[\frac{\beta N_{c}^{2} \omega_{c}^{2}}{4 \alpha}\right]+N_{S} P_{W}}\right)
$$

$$
\mathrm{SC}_{\mathrm{OPC}} \approx \log _{2}\left[1+\frac{\frac{\frac{P}{\omega_{c}}}{1+\gamma_{L}^{2} N_{s}^{2} \Omega_{T}^{2}\left(\frac{P}{2 \pi \omega_{c}}\right)^{2}}}{\frac{N_{S} \gamma_{L}^{2}}{\left[1+\gamma_{L}^{2} N_{s}^{2} \Omega_{T}^{2}\left(\frac{P}{2 \pi \omega_{c}}\right)^{2}\right]^{3}}\left(\frac{P}{\omega_{c}}\right)^{3} \frac{\alpha}{\pi \beta} \ln \left[\frac{N_{c}^{2} \omega_{c}^{2}}{\Omega_{c}^{2}}\right]+N_{S} P_{W}(\omega)}\right]
$$

$$
\mathrm{SC} \approx \log _{2}\left[1+\frac{\frac{P}{\omega_{c}}}{\frac{2 \alpha N_{S} \gamma_{L}^{2}}{\pi \zeta \beta} \frac{\left(\frac{P}{\omega_{c}}\right)^{3}}{\left[1+\gamma_{L}^{2} N_{s}^{2} \Omega_{T}^{2}\left(\frac{P}{2 \pi \omega_{c}}\right)^{2}\right.}}[\right.
$$




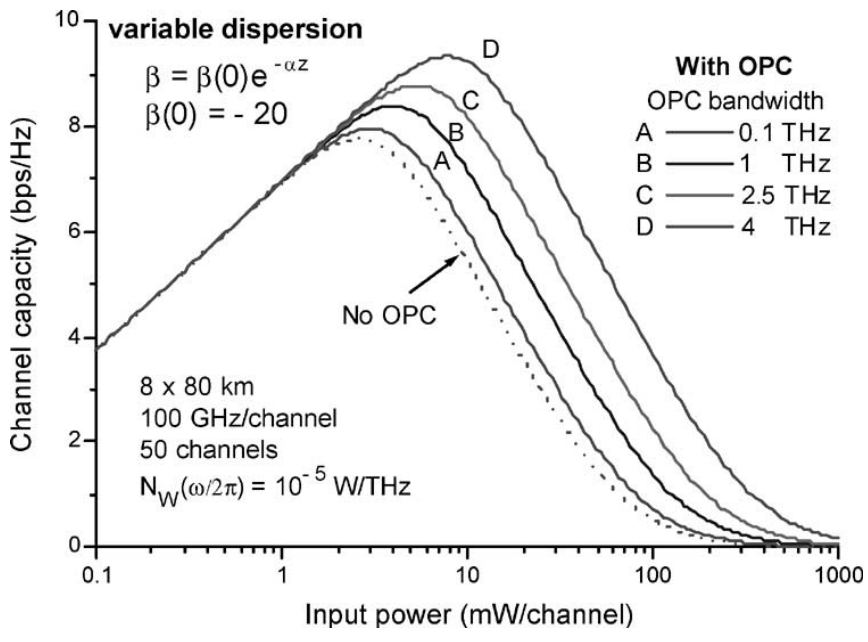

Fig. 1. Effects of a broadband OPC on channel capacity. Significant improvement can be made if the bandwidth of an ideal OPC device is sufficiently wide to cover many channels.

maximum capacity and the optimal operating input power per channel are

$$
\begin{aligned}
& \mathrm{SC}_{\mathrm{MAX}} \approx \log _{2}[ 1+\frac{2}{3} N_{s}^{-1}\left(\gamma_{L} P_{W} \omega_{c}\right)^{-\frac{2}{3}}\left(\frac{4 \alpha}{\pi \zeta \beta \omega_{c}^{2}}\right)^{-\frac{1}{3}} \\
&\left.\times\left(\ln \left(\frac{\zeta \beta N_{c}^{2} \omega_{c}^{2}}{\alpha}\right)-1\right)^{-\frac{1}{3}}\right] \\
& P_{\mathrm{MAX}} \approx\left(P_{W} \omega_{c}\right)^{\frac{1}{3}}\left(\frac{4 \alpha \gamma_{L}^{2}}{\pi \zeta \beta \omega_{c}^{2}}\right)^{-\frac{1}{3}}\left[\ln \left(\frac{\zeta \beta N_{c}^{2} \omega_{c}^{2}}{4 \alpha}\right)-1\right]^{-\frac{1}{3}}
\end{aligned}
$$

Now, we consider nonlinearity precompensation using OPC with a bandwidth of $\Omega_{\mathrm{OPC}}$. The $\mathrm{SC}$ for the central channel is given by

$$
\mathrm{SC} \approx \log _{2}\left[1+\frac{\frac{P}{\omega_{c}}}{2 N_{S}\left(\frac{P}{\omega_{c}}\right)^{3} \frac{\gamma_{L}^{2} \alpha}{\pi \zeta \beta} \ln \left[\frac{N_{c}^{2} \omega_{c}^{2}}{\Omega_{\mathrm{OPC}}^{2}}\right]+N_{S} P_{W}}\right] .
$$

The maximum capacity and the optimal operating input power per channel are

$$
\begin{aligned}
& \mathrm{SC}_{\mathrm{MAX}} \approx \log _{2}[+\frac{2}{3}\left(\sqrt{N_{S}} \gamma_{L} P_{W} \omega_{c}\right)^{-\frac{2}{3}} \\
&\left.\times\left(\frac{4 \alpha}{\pi \zeta \beta \omega_{c}^{2}}\right)^{-\frac{1}{3}}\left(\ln \left[\frac{N_{c}^{2} \omega_{c}^{2}}{\Omega_{\mathrm{OPC}}^{2}}\right]\right)^{-\frac{1}{3}}\right] \\
& P_{\mathrm{MAX}} \approx\left(P_{W} \omega_{c}\right)^{\frac{1}{3}}\left(\frac{4 \alpha \gamma_{L}^{2}}{\pi \zeta \beta \omega_{c}^{2}}\right)^{-\frac{1}{3}}\left(\ln \left[\frac{N_{c}^{2} \omega_{c}^{2}}{\Omega_{\mathrm{OPC}}^{2}}\right]\right)^{-\frac{1}{3}}
\end{aligned}
$$

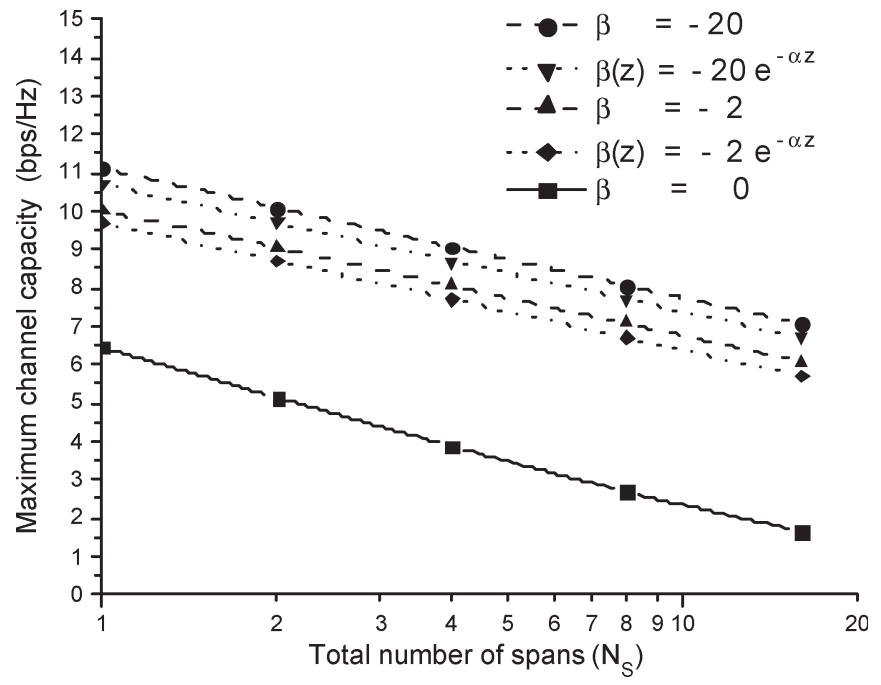

Fig. 2. Maximum channel capacity and its dependence on the total number of spans. Three types of fiber are examined, namely 1) dispersion-free fiber $\beta=0$, 2) variable dispersion fiber $\beta(z)=\beta \exp (-\alpha z)$, and 3) constant dispersion fiber $\beta(z)=\beta$, where $\beta$ is at -2 and $-20 \mathrm{ps}^{2} / \mathrm{km}$. Here, we consider $80 \mathrm{~km} / \mathrm{span}, 100$ channels with $100 \mathrm{GHz}$ channel width, noise power density $P_{W}(\omega / 2 \pi)=10^{-5} \mathrm{~W} / \mathrm{THz}, \alpha=0.048 \mathrm{~km}^{-1}$, and $\gamma=1.22 \mathrm{~km}^{-1} \mathrm{~W}^{-1}$.

\section{Discussions AND CONCLUSION}

We have examined the Shannon channel capacity for three types of optical fibers, namely, 1) with no dispersion, 2) a constant dispersion, and 3) a length-dependent dispersion. A closed-form formula for maximum channel capacity and optimal input power is prescribed in terms of dispersion, number of spans, amplifier noise power, channel band width, etc. The dependence of the maximum channel capacity follows a simple form of $\mathrm{SC}_{\mathrm{MAX}} \sim \log _{2}\left[N_{S}^{-4 / 3}\left(N_{c} \gamma_{L} P_{W}\right)^{-2 / 3}\right]$ for the dispersion-free fiber, but for the fiber with significant dispersion, $\mathrm{SC}_{\mathrm{MAX}} \sim \log _{2}\left[N_{S}^{-1}\left(N_{c} \gamma_{L} P_{W}\right)^{-2 / 3}\right]$. We have also studied the impact of OPC, which reduces degradation caused by interchannel and intrachannel nonlinear effects [5], [6]. The improvement by OPC is illustrated in Fig. 1. The capacity improvement by OPC is relatively small unless the bandwidth of the OPC device comes close to the total spectral width of the transmitted channels. The comparison among the three categories of fibers is illustrated in Fig. 2, showing the maximum channel capacity for these three types of fiber and its dependence on the total number of spans. The presence of a larger dispersion induces bandlimiting filtering that reduces nonlinear noises. For dispersion-free fibers, a Volterra series expansion of all orders is included. The exact maximum channel capacity [2] is similar to the truncation approximation (less than 5\% in difference). For dispersive fibers, truncation to the first order of the series expansion is used. The result in (14) is reasonably accurate at an operating power of $5-10 \mathrm{~mW}$ per 150-GHz-wide channel [4]. So long as one is concerned with the ultimate maximum capacity, which occurs at low power, the truncation is valid. In other treatments of channel capacity [7]-[9], various assumption and approximations were used with regard to modeling noises from self-phase modulation, cross-phase modulation, and four-wave mixing. The maximum channel capacity obtained by different methods is similar in 
magnitude. At a much higher input power, one can no longer classify nonlinear noises into these three types, as the cross terms among them and their correlations become complicated. These various approaches are approximate and meaningful to a certain degree of input power.

In conclusion, the main goal of this study is to obtain simple approximated formulae for channel capacity and to show that the maximum channel capacity is much greater than the current DWDM transmission systems of $1 \mathrm{bit} / \mathrm{s} / \mathrm{Hz}$ at best [6]. A novel paradigm for transmission system design may be needed to reach values closer to the estimated theoretical channel capacity.

\section{ACKNOWLEDGMENT}

The author would like to thank G. J. Foschini, J. Salz, R. E. Slusher, H. Kogelnik, and A. Chraplyvy for initiating the capacity modeling project and many helpful discussions.

\section{REFERENCES}

[1] C. E. Shannon, "A mathematical theory of communication," Bell Syst. Tech. J., vol. 27, no. 3, pp. 379-423, 1948. and 623-656.

[2] J. Tang, "I. The Shannon channel capacity of dispersion-free nonlinear optical fiber transmission. An exact closed-form formula for arbitrary operating power," J. Lightw. Technol., vol. 19, no. 8, pp. 1104-1109, Aug. 2001.

[3] noises on Shannon channel capacity of dispersion-free nonlinear optical fiber transmission. An exact closed-form treatment," J. Lightw. Technol., vol. 19, no. 8, pp. 1110-1115, Aug. 2001.

[4] _ - "The channel capacity of a multispan DWDM system employing dispersive nonlinear optical fibers and an ideal coherent optical receiver," J. Lightw. Technol., vol. 20, no. 7, pp. 1095-1101, Jul. 2002.
[5] S. Watanabe and M. Shirasaki, "Exact compensation for both chromatic dispersion and Kerr effect in a transmission fiber using phase conjugation," J. Lightw. Technol., vol. 14, no. 3, pp. 243-248, Mar. 1996.

[6] A. Chowdhury, G. Raybon, R.-J. Essiambre, J. H. Sinsky, A. Adamiecki, J. Leuthold, C. R. Doerr, and S. Chandrasekhar, "Compensation of intrachannel nonlinearities in 40-Gb/s pseudolinear systems using opticalphase conjugation," J. Lightw. Technol., vol. 23, no. 1, pp. 172-177, Jan. 2005.

[7] P. P. Mitra and J. B. Stark, "Nonlinear limits to the information capacity of optical fiber communication," Nature, vol. 411, no. 6841, pp. 1027-1030, Jun. 2001.

[8] E. E. Narimanov and P. Mitra, "The channel capacity of a fiber optics communication system: Perturbation theory," J. Lightw. Technol., vol. 20, no. 3, pp. 530-537, Mar. 2002.

[9] J. M. Kahn and K. P. Ho, "Spectral efficiency limits and modulation/detection techniques for DWDM systems," IEEE J. Sel. Topics Quantum Electron., vol. 10, no. 2, pp. 259-272, Mar./Apr. 2004.

[10] G. P. Agrawal, Nonlinear Optical Fibers, 2nd ed. New York: Academic, 1995, pp. 49-54.

[11] K. V. Peddanarappagari and M. Brandt-Pearce, "Volterra series transfer function of single mode fibers," J. Lightw. Technol., vol. 15, no. 12, pp. 2232-2241, Dec. 1997.

[12] M. S. Pinsker, Information and Information Stability of Random Variables and Processes. San Francisco, CA: Holden Day, 1964, pp. 160-201.

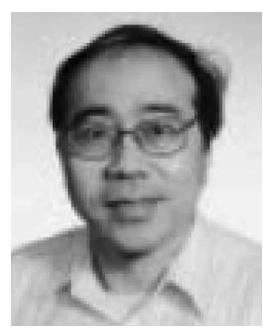

Jau Tang received the B.S. degree from the National Taiwan University, Taipei, Taiwan, R.O.C., in 1974, the M.S. degree from the National Tsing Hua University, Hsinchu, Taiwan, in 1976, and the Ph.D. degree from the University of California, Berkeley, in 1981, all in physics.

$\mathrm{He}$ had been with Argonne National Laboratory, Argonne, IL, Bell Laboratories, Holmdel, NJ, and Opvista, Irvine, CA. He is currently a Senior Scientist at the California Institute of Technology, Pasadena. 